Roenker, D. L. Thompson. C. P. \& Brown. S. C. Comparison of measures for the estimation of clustering in free recall. Psychological Bulletin. 1971.76. 45-48.

Wallace. W. P. Consistency of emission order in free recall. Journal of Verbal Learning \& Verbal Behavior. 1970.9. 58-68.
Wood G. E Underwood. B. J. Implicit responses and conceptual similarity. Journal of Verbal Learning \& Verbal Behatior. 1967. 6. 1-10.

(Received for publication June 18. 1973.)

\title{
Selective attention and dimensional learning: A logical analysis of two-stage attention theories*
}

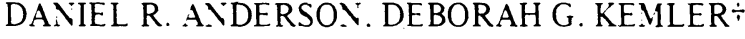 and BRYAN E. SHEPP \\ Brown Lniversit: Pro:idence. R.I. 0.912}

The argument of the present paper is that attention theories (e.g., Sutherland \& Mackintosh, 1972) make logically independent assumptions of selective attention and dimensional learning. The separability of these assumptions is illustrated by a model that assumes dimensional learning but no selective attention. The model successfully predicts the results of discriminative shift studies (e.g., ID vs ED shift comparisons) and supports the conclusion that a selective attention mechanism is not necessary to explain the results of such studies.

Many transfer phenomena in discriminative learning are frequently alledged to support theories of selective attention. Included among these phenomena are dimensional learning (Shepp \& Eimas. 1964). the overlearning reversal effect (Lovejoy. 1966). progressive improvement in serial reversal learning (Mackintosh. 1969). and the execution of reversal shifts in the optional shift paradigm (Eimas. 1969). Other related effects have beeen discussed by Lovejoy (1968).

*Preparation of this paper was supported by Research Grants HD 04320 and $M H 16642$ from the Lnited States Public Health Service. awarded to the third author. and bv Office of Education Grant OEG-1-71-0108508. awarded to the first author. Requests for reprints should be sent to Daniel $R$. Anderson. Department of Psychology. Bartlett Hall. University of IIassachusetts. Amherst. Mass. 01003

+Now at the Eniversity of Pennsvliania.
Sutherland \& Mackintosh (1971). and Fisher \& Zeaman (1972).

The theories in question (Lovejoy. 1968: Fisher \& Zeaman. 1972: Sutherland \& Mackintosh. 1971: Zeaman \& House. 1963) commonly assume that during solution of a discriminative task. attention to a relevant dimension is strengthened while attention to irrelevant dimensions is weakened. Thus. the models assume that Ss learn something about whole dimensions of stimuli in addition to learning something about the specific cues that appear in a single task. With only this assumption of dimensional learning. attentional theories have predicted the phenomena mentioned above and have described some of the conditions which either enhance or constrain the appearance of some phenomena (e.g.. see Lovejoy. 1966). These successes of attention theory have led many investigators to conclude that a selective attention mechanism is responsible for these transfer phenomena and have led still other investigators to argue that some developmental or comparative differences in discriminative performance are due to differences in selective attention mechanisms (e.g.. Tennant \& Bitterman. 1972: Wolff. 1967). In the present paper. however. we suggest that while the results of these various transfer experiments do require some explanation in terms of dimensional control and learning. they do not necessarily imply selective attention.

An examination of two-stage attentional theories reveals at least two fundamental. hut independent. sets 
of assumptions about the processes underlying discriminative learning. One set of assumptions concerns how much is learned in the discriminative situation: these are the assumptions of selective attention. On a single trial. the $\mathrm{S}$ is assumed to be controlled by and to learn about only a subset of the total set of potentially effective stimuli. The second set of assumptions concerns what is learned. During the course of discriminative training. the $S$ presumably acquires not only specific stimulus-response associations, but also differential tendencies to be controlled by whole dimensions of the stimulus. By definition, this dimensional learning is not specific to the cues representing the dimension in the task; rather, it transfers to any new cues on that dimension.

The logical independence of dimensional learning and selective attention assumptions is illustrated in Table 1 by showing representative theories that are committed to particular alternative assumptions. One binary factor denotes whether or not a theory assumes selective attention. while the second and orthogonal factor denotes dimensional vs nondimensional learning. This classification shows some clear differences and similarities between theories. For example. Trabasso \& Bower (1968) and Zeaman \& House (1963) both assume selective attention, in that the $S$ 's choice behavior is selectively controlled and his learning is restricted on a single trial. Yet, Trabasso and Bower assume that all learning is specific to the cues of a given task, whereas Zeaman and House assume dimensional as well as specific cue learning. Spence's classical continuity theory (1936) is like that of Trabasso and Bower in assuming only the learning of specific associations, ${ }^{1}$ but it is different from all attentional models in assuming that the strengths of all components of the chosen stimulus are modified on each trial.

According to this analysis, it is possible to develop a model, defined by the empty cell of Table 1, that assumes no selective attention but allows dimensional learning to occur. In the present paper, a sketch of this type of model and its consequences are provided. 2 The model incorporates the fundamental assumption of any nonattention theory: on every trial, the $S$ learns about and is controlled by all aspects of the stimulus which are effective at the S's receptors. Like Spence's continuity theory (1936), the model assumes that each cue of the total stimulus array acquires, through training, a certain strength (cue strength), i.e., a tendency to elicit an approach response. These cue strengths are modified by the trial outcomes: reinforcement leads to an increase in tha strengths of all cues in the stimulus to which the response was directed. Nonreinforcement results in a decrease in the strengths of all cues in the stimulus that was chosen. At all times, the strengths of the pair of cues on any dimension sum to 1.00 , so that the strengths of the cues are bounded between 0.00 and 1.00, and are dependent within any one dimension.

A novel feature of the present nonattention model is
Table 1

Classification of Theories of Discriminative Learning

Selective Attention No Selective Attention

Dimensional Learning

Zeaman \& House (1963)

Lovejoy (1966)

Lovejoy (1968)

No Dimensional Learning

Restle (1962)

Bower \& Trabasso (1964)

Spence (1936)

Trabasso \& Bower (1968)

Burke \& Estes (1957)

the following assumption: that each dimension of the total stimulus array acquires, through training, a "control strength" which depends on the consistency of reinforcement of its cues. If the strength of Cue Al is greater than or equal to the strength of Cue A2 on a particular trial, and if the outcome on that trial is consistent with the direction of these strengths (i.e., choice of the stimulus containing Al is reinforced or choice of the stimulus containing $\mathbf{A} 2$ is nonreinforced), then the control strength of Dimension A increases. If, on the other hand, the outcome of that trial is inconsistent with the relative strengths of the cues on Dimension A, then the control strength of the dimension decreases. The control strength of any dimension may take on values between 0.00 and 1.00. Like all cue strengths, all dimensional control strengths are modified on every trial; thus, the assumption of totally nonselective learning is not violated.

The response rule for the model is quite straightforward. On each trial of a simultaneous discrimination task, each of the two multidimensional stimuli has a net strength which depends on the strengths of all its composite cues, each weighted by the control strength of the dimension along which it lies. Specifically, the net strength of each multidimensional stimulus is computed as the sum of the products of cue strengths and their appropriate dimensional control strengths. The probability that $\mathrm{S}$ chooses one or the other stimulus is directly proportional to the relative net strength of that stimulus, according to Luce's choice axoim (Luce, 1959).

The model arranges that as the strengths of positive and negative cues consistently diverge over trials of training, the control strength of the relevant dimension continuously increases. Furthermore, as the strengths of irrelevant cues fluctuate over trials due to inconsistent reinforcement, the control strengths of irrelevant dimensions tend to decrease. The assumption is now added that control strengths modified by training in one task transfer to a second task in which the dimensions are represented by new cues.

With only these assumptions, the model can predict performances in situations where dimensions from one task are retained in a second. To illustrate, consider the intradimensional vs extradimensional shift paradigm. Ss are first trained on a discrimination in which one 
dimension re.g. form) is relevant and a second re.g. olor) is irrelevant. Then a second task is presented. For Ss given an intradimensional shift. form remains relevant: for Ss given an extradimensional shift. color becomes relevant. Since the specific cues of the initial task are replaced by others in transfer. cue strengths do not contribute to performence in the shift tasks. However. control strengths established in the initial task transfer positively to an intradimensional shift and negatively to an extradimensional shift. A simulation of the model with eight stat-Ss in each shift condition revealed that ID Ss learned with a mean trial of last error equal to 0.4 as compared with a mean TLE of 38.0 for ED Ss. This difference in learning rate is significant $(p<.01)$. and the performances of the stat-Ss compare favorably with the performances of real Ss (see. e.g.. Kemler \& Shepp. 1971). Simulations of the model also vield an overlearning reversal effect.

It is. of course. critical to the arguments of this paper that the reader be convinced that the proposed model makes no assumptions of selective attention. Certainly. it will be agreed that the model does not allow selective learning: The strengths of all cues and of all dimensions are affected by the reinforcement outcome on every trial. It may be less clear. however. that assumptions of selective attention are not implicit in the model's rule for response decermination. Recall that the choice on each trial is generated by summing for each multidimensional stimulus the net strengths of its component cues. each first weighted by the control strength of the dimension along which it lies. Thus. the cues of dimensions with high control strengths might be said to be "selectively" prepotent in determining the choice response. However. if. simply by rirtue of allowing prepotency in response determination. the present model is classified as attentional. then so also must Spence's 1936 formulation (ordinarily the classic example of noncontinuity or nonattention theory) be considered attentional. thus rendering the description totally meaningless. Both models arrange that as the learning process proceeds. the relevant cues gain prepotent control of the response. but no active intervention of a selective mechanism is presumed at any stage of the learning process.

\section{DISCUSSION}

We have chosen not to explore the possibilities of the present nonattentional model much beyond the depth of this report. The reader may justifiably question the wisdom of this choice. for it is not possible to determine how well the model would deal with other phenomena of discriminative learning. Howerer. we hesitate to add still another full-blown multiparametered model to the numerous alternatives that hate recently appeared in the discrimination learning literature (I isher \& Zeaman. 1972: Lovejo!. 1968: Spiker. 1970: Sutherland \& Yackintosh. 1971). To decide between these model requires considerably more empirical work in the area if the number of phenomena to be explained are to exced appreciably the number of parameters that are free to vars. We have shown previously i.Anderion. 1972: Kemler \& Anderson. 1972: Kemler \& Shepp. 1971: Shepp.
Kemler d Andeson. 19-2) that dicriminative learning model that awume vers different learning and attentional mechanism often make identical prediction in a variet! of experimental situations. Therefore. more well-considered analyses and spevifi tests of the alternatis assumption of discriminative learning models are in order. Ai we have demonstrated here. dimensional transfer paradigms do no: provide a test of selective attentionai mechanisms.

\section{REFERENCES}

Anderson. D. R. The effects of prior training on redundancy learning in children. Journal of Experimental Child Psychology. 1972, in press.

Bower, G. H., \& Trabasso, T. Concept identification. In R. C. Atkinson (Ed.). Studies in mathematical psychology. Stanford: Stanford University Press, 1964. Pp. 32-94.

Burke. C. J.. \& Estes. W. K. A component model for stimulus variables in discrimination learning. Psychometrika. 1957, 22. $133 \cdot 145$.

Eimas. P. D. Attentional processes in optional shift behavior. Journal of Comparative \& Physiological Psychology. 1969.69. $166 \cdot 169$.

Fisher U. A. \& Zeaman. D. An attention-retention theory of retardate discrimination learning. In $N$. $R$. Ellis (Ed.). Inteinational retieu of research in mental retardation. Vol. 8. New York: Academic Press. 1972

Kemler. D. G.. \& Anderson. D. R. The breadth of attention in learning: A new one-look model. British Journal of Mathematical \& Statistical Psychology. 1972, in press.

Kemler. D. G.. \& Shepp, B. E. Learning and transfer of dimensional relevance and irrelevance in children. Journal of Experimental Psychology. 1971.90.120-127.

Lovejoy E Analysis of the overlearning reversal effect. Psychological Reriew. 1966, 73, 87-103.

Lovejov. E. Attention in discrimination learning. San Francisco: Holden-Day, 1968.

Luce. R. D. Individual choice behatior: A theoretical analysis New York: Wiley. 1959

Mackintosh. $\therefore$. J. Comparative studies of reversal and probability learning: Rats. birds and fish. In R. M. Gilbert and $\mathrm{N}$. S. Sutherland (Eds.). Animal discrimination learning. London: Academic Press. 1969.

Restle, $F$. The selection of strategies in cue learning. Psychological Review, 1962, 69, 11-19.

Shepp. B. E.. \& Eimas. P. D. Intradimensional and extradimensional shifts in the rat. Journal of Comparative \& Physiological Psychology, 1964. 57. 357-361.

Shepp. B. E.. \& Howard. D. V. Are differential orienting responses necessary for dimensional learning and transfer? Journal of Experimental Psychology. 1973. in press.

Shepp. B. E.. Kemler. D. G.. \& Anderson. D. R. Selective attention and the breadth of learning: An extension of the one-look model. Psychological Review. 1972. 79. 317-328.

Spence. K. W. The nature of discrimination learning in animals. Psychological Review. 1936.43.427-449.

Spiker. C. C. An extension of Hull-Spence discrimination learning theory. Psychological Reivew. 1970.77. 496-515.

Sutherland. $X$. S.. \& Mackintosh. X. J. Mechanisms of animal discrimination learning. New York: Academic Press. 1971.

Trabasso. T.. \& Bower. G. H. Attention in learning. Few York: Wilev. 1968

Tennant. W. A.. \& Bitterman. M. E. Intradimensional and extradimenaional transfer in the goldfish. Paper presented at the Psychonomic Snciety Annual Meeting in St. Louis. Mo. November 1972

Wolff. J. L Concept-shift and discrimination-reversal learning in humans. Psychological Bulletin. 1967.68. 369-408

Zeaman. D.. \& House. B. J. The role of attention in retardate discrimination learning. In $N$. R. Ellis (Ed.). Handbook of mental deficienc. Psychological theory and research. New York: McGraw-Hill. 1963. Pp. 159-223.

\section{NOTES}

1. We recognize that the $S-R$ theories can predict performances in transfer by principles of stimulus generalization. However. Shepp \& Howard (1973) have shown that generalization does not contribute significantly to such phenomena as dimensional learning.

2. Details of the model and the results of simulations can be obtained from B. E. Shepp. Department of Psichology. Brown Eniversity. Providence. R. I. 02912.

$$
\text { 1 Recoived for puhlication lal! 9. 1473.1 }
$$

